\title{
The model of the influence for the public interests based on Markov chain
}

\author{
Xiao-Jian Lu
}

\author{
Department of Power Engineering, North China Electric Power University, Baoding, Hebei, P.R. \\ China
}

Keywords: Information Network, Markov Model

\begin{abstract}
In this paper, the Markov model is built to explore how social information spreads and works, especially through the networks. the note distance calculation model based on Cluster analysis is proposed to determine the factors affecting public interest and opinions. We divide people in a region into three types according to their interest and opinions, and build up a simplified information network, assuming that the public interest and opinions just depend on current conditions. In this model, Markov model is used to determine the percentage of each kind of people. It is shown that, as the distance of the nodes changes, the final percentage of the three types of people will be also changed. To verify the effectiveness and sensitivity, we apply our model to a specific area, which shows a satisfactory result.
\end{abstract}

\section{Introduction}

Social networks are information networks that describe how individuals or entities interact with one another. With the constantly development of science and technology, information networks also evolve. Several decades ago, newspapers and radios are the public's top daily news source. In recent years, more people get news from televisions, internet and telephones. Due to the expanding ways to get news, information spreads further and wider, and it plays a more and more important role in our society. We can acquire information rapidly and disseminate it. Therefore, it is significant to study on the evolution and influence in society's information networks.

In this paper, we will explore society's information networks from two sides: one is the information spread; two is the change of public opinion with the development of information network. In terms of the first problem, we establish two models to analyze information value as well as its source. For the second, we construct a simplified information network to obtain affecting factors affecting public opinion.

\section{The Affecting Factors of Public Interest and Opinions}

The model.

Although the media of information develop rapidly, the information spread boils down to human-to-human communication. So we set a model about information spread among people in a region.

- Step 1. Dividing the People

We divided people in a region into three types according to their interest or opinions by means of Cluster analysis: A, B and C. A and C are opposites, while B is between A and C. The topology of information network is simplified as shown in fig.1. 


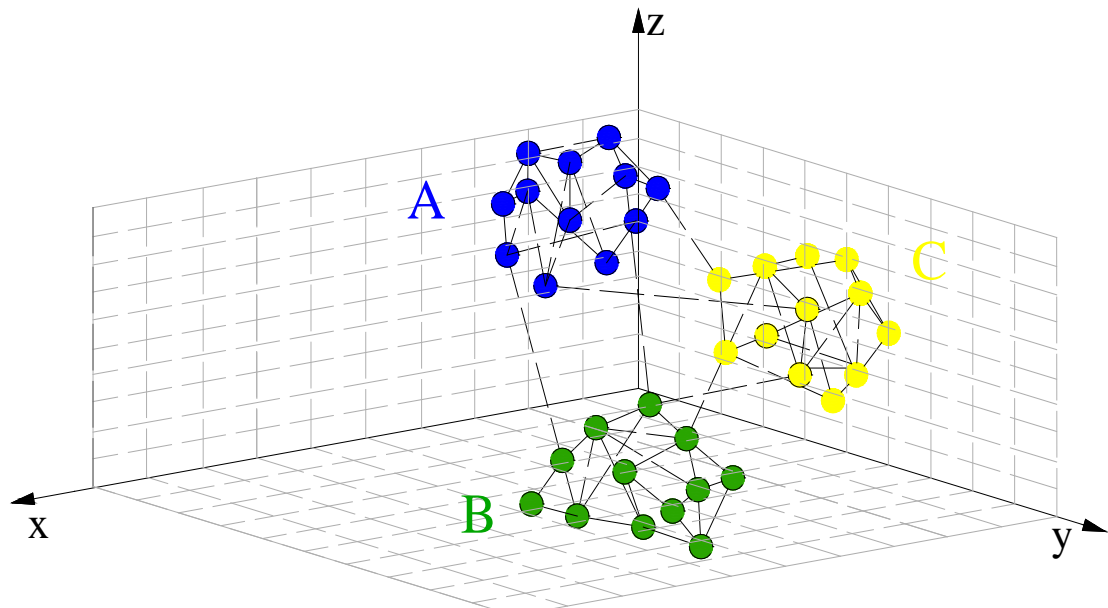

Step 2. Markov Chain

Fig.1 a simplified information network

Assume that the public interest and opinions just depend on current conditions. So we apply Markov Chain to this problem. The nodes in fig. 1 represent the people. The closer the nodes are, the easier his/her interest or opinions are changed by around people. So the probability of the interest or opinions change can be derived by distance. The distance is shown as tab.1.

Tab.1 The distance between two types of nodes

\begin{tabular}{|c|c|c|c|}
\hline & $A$ & $B$ & $C$ \\
\hline$A$ & $D_{A}$ & $D_{A B}$ & $D_{A C}$ \\
\hline$B$ & $D_{B A}$ & $D_{B}$ & $D_{B C}$ \\
\hline$C$ & $D_{C A}$ & $D_{C B}$ & $D_{C}$ \\
\hline
\end{tabular}

The probability can be expressed as the following State Transition Matrix:

$P=\left[\begin{array}{ccc}p_{A} & p_{A B} & p_{A C} \\ p_{B A} & p_{B} & p_{B C} \\ p_{C A} & p_{C B} & p_{C}\end{array}\right]$

Where:

$p_{A}=k_{A} \cdot \frac{1}{D_{A}}, \quad p_{A B}=k_{A} \cdot \frac{1}{D_{A B}}, \quad p_{A C}=k_{A} \cdot \frac{1}{D_{A C}}$,

$p_{A B}=k_{B} \cdot \frac{1}{D_{A B}}, p_{B}=k_{B} \cdot \frac{1}{D_{B}}, p_{B C}=k_{B} \cdot \frac{1}{D_{B C}}$,

$p_{C A}=k_{C} \cdot \frac{1}{D_{C A}}, \quad p_{C B}=k_{C} \cdot \frac{1}{D_{C B}}, p_{C}=k_{C} \cdot \frac{1}{D_{C}}$,

$p_{A}+p_{A B}+p_{A C}=1, p_{B A}+p_{B}+p_{B C}=1, p_{C A}+p_{C B}+p_{C}=1$.

Since $D_{i j}=D_{j i}, p_{i j}=p_{j i}$.

- Step 3. The percentage of the three types of people

According to the theory of Markov Chain, we have

$M \cdot P=M$,

where $M$ is the final percentage of the three kinds of people. Solve the equation, we get

$M=\left(m_{A}, m_{B}, m_{C}\right)$.

If the topology changes, we know the final percentage of the three kinds of people will change. It is represented as

$M^{\prime}=\left(\begin{array}{lll}m_{A}^{\prime}, & m_{B}^{\prime}, & m_{C}^{\prime}\end{array}\right)$ 
Comparing $M^{\prime}$ ' with $M$, we can get that if $M^{\prime}$ is different from $M$, the change of topology of the information network can influence the public opinion and interest.

\section{Numerical Simulation}

We apply the model to a specific area. Change the topology information by changing the distance. Choose some people who have different opinion or interest randomly. The fig. 2 shows the three types of people communicate with increasing frequency. Then calculate the percentage of people under three-dimensional system of coordinate.
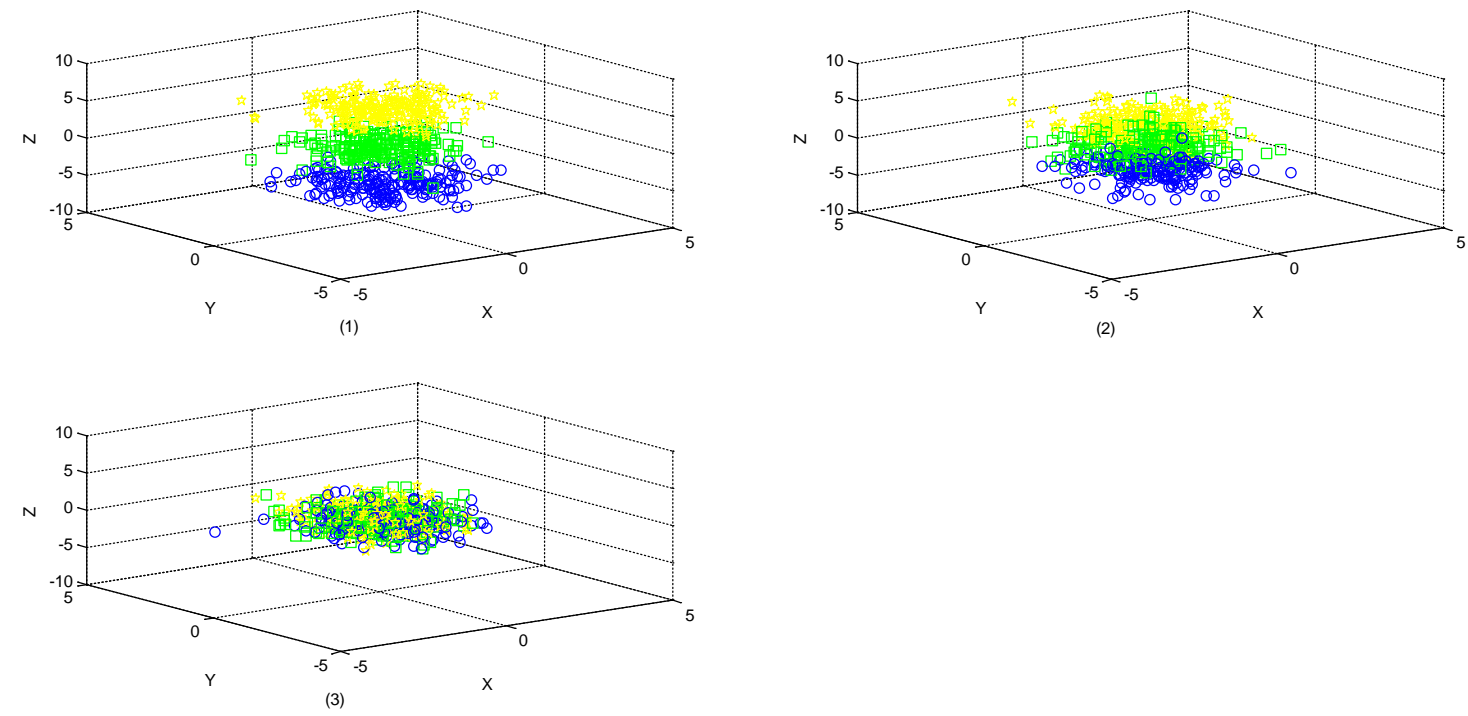

Fig.2 The three situations of distribution

The numerical results are shown as follows.

$$
\begin{aligned}
& P_{(1)}=\left[\begin{array}{lll}
0.3074 & 0.3463 & 0.3463 \\
0.3436 & 0.3128 & 0.3436 \\
0.3471 & 0.3471 & 0.3058
\end{array}\right], M_{(1)}=\left[\begin{array}{lll}
0.3327, & 0.3353, & 0.3319
\end{array}\right] \text {, } \\
& P_{(2)}=\left[\begin{array}{lll}
0.3034 & 0.4657 & 0.2329 \\
0.3818 & 0.2364 & 0.3818 \\
0.2356 & 0.4711 & 0.2934
\end{array}\right], M_{(2)}=\left[\begin{array}{lll}
0.3074, & 0.3834, & 0.3092
\end{array}\right] \text {, } \\
& P_{(3)}=\left[\begin{array}{lll}
0.1760 & 0.5493 & 0.2747 \\
0.4342 & 0.1317 & 0.4342 \\
0.2769 & 0.5538 & 0.1693
\end{array}\right], M_{(3)}=\left[\begin{array}{lll}
0.3001, & 0.3998, & 0.3001
\end{array}\right] \text {. }
\end{aligned}
$$

For different topology of information network, the distribution of three types of people is different. The numerical simulation results show that people communicate more frequently, the percentage of $\mathrm{B}$ increases. In conclusion, the topology of information network can affect public opinions and interest.

\section{Sensitivity Analysis}

We make a sensitivity analysis via MATLAB. We take the percentage of the people of type A. we can see from fig. 3 that when the distance changes, the change of percentage of type A is sensitive. 


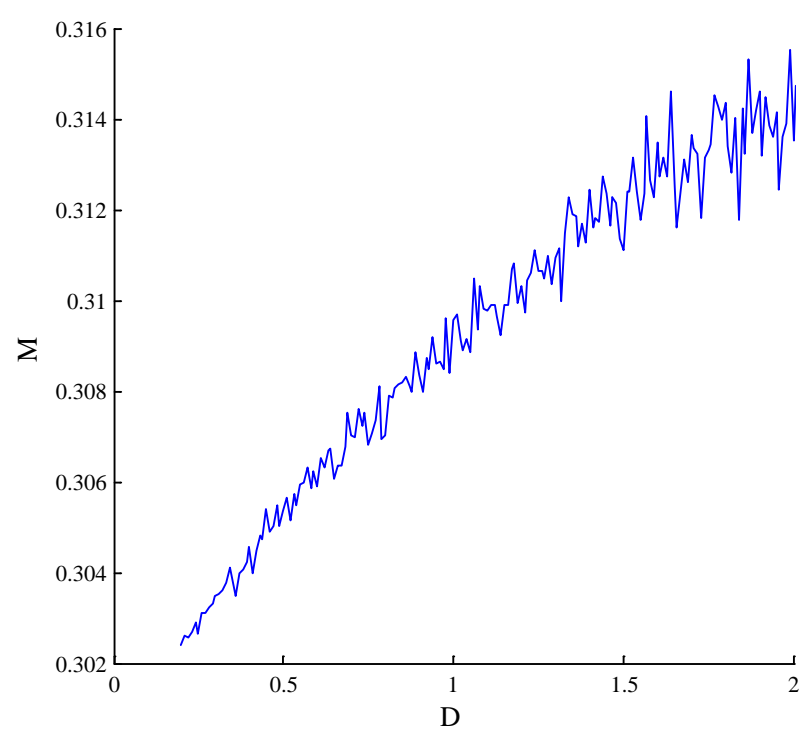

Fig.3 The change of the people A with distance

\section{Summary}

Combining above models, we can know:

Information value and its source affect information spread: (1) If the information has more value, its maximum of spreaders is bigger. (2) Large effect of information source benefits to the spread of information;

The topology of information network influences public opinion and interest.Hence, we determine the affecting factors of information spread and public opinion.

\section{References}

[1] Jeonghwan Jin,Rothrock, L.,McDermott, P.L.,Barnes, M.,Using the Analytic Hierarchy Process to Examine Judgment Consistency in a Complex Multiattribute Task. 2010, pp.1105-1115.

[2] Weijun Hong,Zhipeng Gao,Yuwen Hao,Xiaoxue Li, A novel SCNDR rumor propagation model on online social networks. 2015, pp.154-155.

[3] Decai, Huang; Liangzhong, Shen, "New method for constructing comparison matrix based on the proportion scales in the AHP," in Systems Engineering and Electronics, Journal of , vol.14, no.3, pp.8-13, Sept. 2003

[4] Azni, A.H.; Ahmad, R., Mohamad Noh, Z.A., Correlated node behavior in wireless ad hoc networks: An epidemic model.2012, pp.403-410. 